BULL. AUSTRAL. MATH. SOC.

VOL. $20(1979), 161-163$.

\title{
The inverse of a certain block matrix
}

\section{V.N. Singh}

A simple formula for the inverse of a block matrix with non-zero blocks in the principal diagonal and the first sub-diagonal only is proved. The matrix had arisen in an investigation of a difference equation.

During an investigation of the general homogeneous linear difference equation

$$
\sum_{s=0}^{r} a_{s}(n) u_{n-s}=0, n \geq r,
$$

with $a_{0}(n) \neq 0$ for all $n \geq r$, it was found [2, equation (6)] that the solution involved the inverse of a non-singular block lower triangular matrix of the following type

$$
A_{(N)}=\left[\begin{array}{ccccccc}
A_{1} & 0_{r} & 0_{r} & \cdots & 0_{r} & 0_{r} & 0_{r, s} \\
B_{2} & A_{2} & 0_{r} & \ldots & 0_{r} & 0_{r} & 0_{r, s} \\
0_{r} & B_{3} & A_{3} & \ldots & 0_{r} & 0_{r} & 0_{r, s} \\
\cdots & \ldots & \ldots & \ldots & \ldots & \ldots & \ldots \\
0_{r} & 0_{r} & 0_{r} & \ldots & B_{N-1} & A_{N-1} & 0_{r, s} \\
0_{s, r} & 0_{s, r} & 0_{s, r} & \ldots & 0_{s, r} & B_{N} & A_{N}
\end{array}\right] .
$$

Here $N$ is the integral part of $n / r$, and ${ }_{p}, \dot{q}$ denotes the null matrix of dimension $p$ by $q$ with $o_{r, r} \equiv o_{r}$; the matrices $A_{k}, B_{k}$ have the

Received 9 January 1979. 
order $r$ for $k=1, \ldots, N-1$, while the dimensions of $A_{N}$ and $B_{N}$ are $s$ by $s$ and $s$ by $r$ respectively with $l \leq s \leq r$.

In this note, we prove that the above matrix $A_{(N)}$ has the following inverse:

$$
A_{(N)}^{-1}=\left[L_{i j}\right], i, j=1, \ldots, N
$$

where

$$
\begin{aligned}
& L_{i i}=A_{i}^{-1}, \quad i=1, \ldots, N, \\
& L_{i j}=o_{r}, i<j \leq N-1, L_{i N}=O_{r, s}, \quad 1 \leq i<N,
\end{aligned}
$$

and

$$
L_{i j}=(-1)^{i+j}\left\{\sum_{k=i}^{j+1}\left\{A_{k}^{-1} B_{k}\right)\right\} A_{j}^{-1}, i=2, \ldots, N, j=1, \ldots, i-1 .
$$

The proof is by induction on $N$. For $N=2$, formula (1) takes the form

$$
A_{(2)}^{-1}=\left[\begin{array}{cc}
A_{1}^{-1} & 0_{r, s} \\
-A_{2}^{-1} B_{2} A_{1}^{-1} & A_{2}^{-1}
\end{array}\right] \text {, }
$$

which is a special case of a well-known result $[1$, p. 109].

Suppose that (I) holds for a block matrix of order $m$; that is, for $N=m$. Then, for a matrix of order $m+1$, by (2) we have

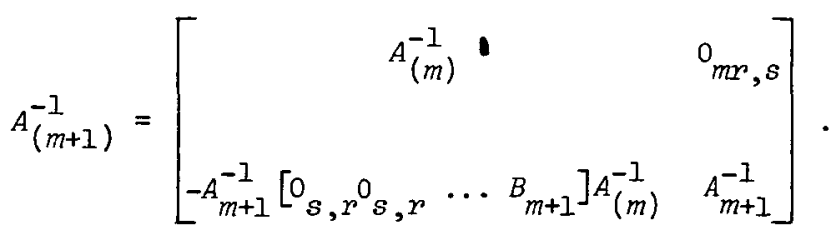

Since

$$
-A_{m+1}^{-1}\left[{ }_{s, r}{ }^{0} s, r \cdots B_{m+1}\right] A_{(m)}^{-1}=-A_{m+1}^{-1} B_{m+1}\left[L_{m 1} L_{m 2} \cdots L_{m m}\right]
$$

it is easy to see that, on account of the induction hypothesis, (1) holds for $N=m+I$. The proof of (I) is thus complete. 


\section{References}

[1] George F. Hadley, Linear algebra (Addison-Wesley, Reading, Massachusetts; London; 1961).

[2] V.N. Singh, "Solution of a general homogeneous linear difference equation", submitted.

Department of Mathematics and Astronomy, University of Lucknow,

Lucknow,

India. 